\title{
SIRT3 Protects Against Cognitive Dysfunction Induced by Sepsis-associated Encephalopathy Via JNK/p66Shc-Regulated Mitochondrial Apoptosis in Mice
}

\section{Zhuochen Lyu}

Shanghai Jiao Tong University Medical School Affiliated Ruijin Hospital

Shiyuan Luo

Shanghai Jiao Tong University Medical School Affiliated Ruijin Hospital

Yinjiao Li

Shanghai Jiao Tong University Medical School Affiliated Ruijin Hospital

Liangfang Yao

Shanghai General Hospital

\section{Feng Chen}

Shanghai General Hospital

Qing Tu

Shanghai General Hospital

\section{Yuan Yuan}

Shanghai General Hospital

Jinbao Li

Shanghai General Hospital

\section{Yan Luo ( $\nabla$ ly11087@rjh.com.cn )}

Shanghai Jiao Tong University Medical School Affiliated Ruijin Hospital https://orcid.org/0000-00030431-3162

\section{Research}

Keywords: SIRT3, sepsis-associated encephalopathy, cognitive dysfunction, mitochondrial apoptosis

Posted Date: December 8th, 2021

DOI: https://doi.org/10.21203/rs.3.rs-895666/v2

License: (c) (i) This work is licensed under a Creative Commons Attribution 4.0 International License. Read Full License 


\section{Abstract}

Background: Sepsis-associated encephalopathy (SAE) is one of the severe central nervous system complications. Oxidative stress and synaptic dysfunction were involved in cognitive impairment induced by SAE. The mitochondrial nicotinamide adenine dinucleotide $\left(\mathrm{NAD}^{+}\right)$dependent deacetylase, sirtuin3 (SIRT3), plays a critical role in regulating mitochondrial function. The aim of this study was to evaluate the effect of SIRT3 in cognitive dysfunction induced by SAE.

Methods: Mice were treated with lipopolysaccharide (LPS, $10 \mathrm{mg} / \mathrm{kg}$, i.p.). Contextual and cue memory were evaluated by fear conditioning test in wild-type (WT) and SIRT3-deficient (SIRT3-/-) mice. Synapseassociated proteins and mitochondrial apoptosis-associated protein were examined by western blotting. In vitro studies, acetylation levels of cyclophilin D (CypD) were detected with different SIRT3 deacetylase activity in HT22 cells after LPS-induced microglia supernatant (Mi-sup) exposure. Oxidative stress was detected by reactive oxygen species (ROS) staining, and mitochondrial membrane potential (MMP) was detected by JC-1 staining, and mitochondrial membrane permeability transition pore (MPTP) opening was detected by Calcein and $\mathrm{Co}^{2+}$ staining. Furthermore, the phosphorylation levels of mitochondrial p66Shc and JNK were evaluated by western blotting.

Results: SIRT3 expression was diminished in hippocampus of mice after LPS treatment. SIRT3-deficiency contributed to more severe contextual memory loss and synaptic dysfunction, decreased ratio of Bcl2/Bax and increased Cyt $C$ release to cytoplasm in hippocampus compared with wild-type controls. In HT22 cells, lysine acetylation levels of CypD were significantly increased after Mi-sup exposure and further enhanced with 3-TYP (SIRT3 deacetylation inhibitor) pretreatment, in association with the accumulation of ROS, declined MMP and increased MPTP opening, as well as the increased mitochondrial Cyt $\mathrm{C}$ release and phosphorylation levels of mitochondrial JNK and p66Shc-Ser36. SIRT3 overexpression restored CypD lysine acetylation levels and MPTP opening in HT22 cells after Mi-sup exposure and reduced mitochondrial JNK and p66Shc activation.

Conclusions: Taken together, our results showed that SIRT3-mediated CypD deacetylation was involved in LPS-induced hippocampal synaptic dysfunction, via ROS accumulation, declined MMP, increased MPTP opening, mitochondrial Cyt $\mathrm{C}$ release and mitochondrial apoptosis of hippocampal neuron via JNK/p66Shc pathway. Our results revealed that SIRT3 may be a promising therapeutic and diagnostic target for cognitive dysfunction induced by SAE.

\section{Background}

It is universally acknowledged that severe sepsis is related to multi-system damage including brain dysfunction, and sepsis-associated encephalopathy (SAE) is one of the severe central nervous system complications in patients in intensive care units for its cognitive dysfunction [1, 2]. SAE is associated with increased morbidity and mortality, and it can influence the short-term outcome and long-term recovery [3]. 
Oxidative stress and apoptosis in hippocampal neurons have been reported to involve in cognitive impairment in SAE $[4,5]$.

One of the most important pathogenesis of $\operatorname{SAE}$ is mitochondrial dysfunction $[6,7,8]$. Mitochondria is reported to be the major source of reactive oxygen species (ROS) and initiation of apoptosis $[9,10,11]$. Normal mitochondria produce ATP to support cellular metabolism, whereas damaged mitochondria release pro-apoptotic factors to initiate programmed cell death. Several studies found that inflammation reduces mitochondrial membrane potential and increases oxidative stress [12,13], however, the mediator of these inflammation-induced pathological alterations in the mitochondria has not been clearly identified.

Sirtuin 3 (SIRT3), a member of nicotinamide adenine dinucleotide $\left(N A D^{+}\right)$dependent deacetylases, has been identified as a key regulator of mitochondrial function in sepsis-induced multiple organ failure, including lung [14], heart $[15,16]$ and kidney $[17,18]$. Cyclophilin D (CypD) is the key component of mitochondrial membrane permeability transition pore (MPTP) and acetylation of CypD leads to an increased susceptibility to MPTP opening $[19,20,21,22]$. Study showed that SIRT3-mediated CypD deacetylation attributed to the MPTP opening in lipopolysaccharide (LPS)-induced endothelial barrier disruption [23]. However, the underlying mechanisms by which SIRT3-mediated CypD deacetylation in LPS-induced mitochondrial dysfunction in hippocampus have not been well recognized.

Several studies showed that $66 \mathrm{kDa}$ Src homology 2 domain-containing protein (p66Shc) plays a specific role in mitochondrial ROS generation and translates oxidative stress signals into cell apoptosis [24, 25]. Modified p66Shc could translocate into the mitochondria and oxidizes cytochrome $\mathrm{C}$ (Cyt $\mathrm{C}$ ) to yield $\mathrm{H}_{2} \mathrm{O}_{2}$, which in turn initiates cell apoptosis [26, 27]. However, the effects of p66Shc on sepsis-induced mitochondrial dysfunction in central nervous system are largely unknown, and whether SIRT3 could interact with p66Shc in the process of mitochondrial dysfunction were still unexplored. In the present study, we investigated the effects of SIRT3-mediated cognitive impairment induced by SAE and the following mitochondrial mechanism.

\section{Materials And Methods}

\section{Animals}

Procedures involving animals were approved by the Animal Experimentation Ethics Committee of Ruijin Hospital, and were performed strictly according to the National Institutes of Health Guidelines for the Care and Use of Laboratory Animals. Male wild-type (WT) and SIRT3-knockout (SIRT3-/-) mice were used for the study. The mice were kept on a 12-hour light/dark cycle with free access to food and water.

\section{SAE mouse model}

SAE was induced in mice between 6 to 8-week-old by intraperitoneal (i.p.) administration of $10 \mathrm{mg} / \mathrm{kg}$ LPS (Escherichia coli, serotype 0111: B4, catalog L2630) from Sigma-Aldrich (St. Louis, MO, USA), similar 
to previous studies [50,51, 52]. Animals were divided into 4 groups ( $n=8$ each): WT Control (WT Con) group, WT LPS group, SIRT3-/-Control (SIRT3-/-Con) group and SIRT3/-LPS group. Mice were euthanized 24 hours after LPS administration. The hippocampal tissues were isolated and stored at -80 ${ }^{\circ} \mathrm{C}$ for further analysis.

\section{Behavioral tests}

Behavioral tests included the open field test (OFT) and fear conditioning test (FCT). Spontaneous locomotor activity of the mice in OFT was evaluated as previously described [53]. The apparatus is made up of a white polyester resin chamber $(40 \mathrm{~cm}$ x $40 \mathrm{~cm}$ x $40 \mathrm{~cm})$. Mice were placed in the center of the arena and allowed to explore for $300 \mathrm{~s}$, and the total distance moved and the time spent in the center were recorded. The chamber was cleaned with $75 \%$ ethanol after each trail.

The FCT was performed following the protocol as previously described $[54,55]$. On the day of training, mice were placed into an enclosed training chamber and allowed to explore for $180 \mathrm{~s}$. Mice were then exposed to a tone (30 s, $70 \mathrm{~dB}, 3 \mathrm{kHz})$, followed by a $2 \mathrm{~s}$ foot shock $(0.45 \mathrm{~mA})$. Afterward, mice were left in the chamber for additional $30 \mathrm{~s}$. The training period was repeated 5 times. 24 hours after the training session, mice were re-exposed in the same chamber for $300 \mathrm{~s}$ for the contextual fear conditioning test (a hippocampus-dependent task). 4 hours later, mice were placed into a novel chamber which was different in shape, color, and smell from the original chamber for the cued fear conditioning test (a hippocampusindependent task). After $180 \mathrm{~s}$ exploratory period, a training tone (30 s, $70 \mathrm{~dB}, 3 \mathrm{kHz}$ ) was applied for another $30 \mathrm{~s}$ and repeated 4 times at an interval of $40 \mathrm{~s}$, and the time of freezing was recorded. The chamber was cleaned with $75 \%$ ethanol after each trial.

\section{Nissl's staining}

Mice were anesthetized and transcardially perfused with pre-cooled heparinized physiological saline and $4 \%$ paraformaldehyde. After dehydration with gradient ethanol elution and cleared in xylene, cerebral tissues were sliced into $10 \mu \mathrm{m}$ coronal sections using a cryostat (Leica CM1860 UV, Leica) and stored at $-20{ }^{\circ} \mathrm{C}$. Nissl staining (Beyotime Institute of Biotechnology, Shanghai, China) was conducted according to the manufacturer's instructions. In brief, the sections were soaked in $1 \%$ toluidine blue for 5 min at $50{ }^{\circ} \mathrm{C}$, washed by double distilled water, and dehydrated with gradient ethanol elution. The slice sections were observed under a fluorescent microscope.

\section{TUNEL staining}

The TdT-mediated dUTP Nick-End Labeling (TUNEL) staining was used to evaluate neuronal apoptosis in the hippocampus. In Situ Apoptosis Detection Kit was used with manufacturer's instruction (Beyotime Institute of Biotechnology, Shanghai, China). The nuclei were stained with DAPI, and the fluorescent microscopy was used to evaluate neuronal apoptosis.

\section{Immunofluorescence staining}


The brain tissues were stored in $4 \%$ paraformaldehyde and sectioned at $30 \mu \mathrm{m}$, and immunofluorescence staining for SIRT3, NeuN and DAPI were performed. The sections were incubated with SIRT3 antibodies (catalog D22A3, Cell Signaling Technology, Danvers, MA, USA) and NeuN (catalog 66836-1-Ig, ProteinTech Group, Chicago, IL, USA) overnight at $4{ }^{\circ} \mathrm{C}$. Anti-rabbit (catalog 147626) and anti-mouse (catalog 146552) secondary antibodies from Jackson ImmunoResearch were added and incubated for 2 $\mathrm{h}$ at $37^{\circ} \mathrm{C}$, and sections were then washed three times with PBST. After final washing, sections were protected with coverslips, with the nucleus visualized with DAPI (catalog C1005, Beyotime Institute of Biotechnology, Shanghai, China). The brain tissues were observed and analyzed using fluorescence microscope.

HT22 cells were fixed in 4\% paraformaldehyde in PBS for 15 min at room temperature. Cells were then permeabilized in $0.1 \%$ Triton X-100 in PBS for 15 min and blocked with $10 \%$ donkey serum in PBS for $1 \mathrm{~h}$. The culture dishes were then incubated with anti-SHC phosphor $\mathrm{S} 36$ (catalog 54518 , Abcam, Cambridge, UK) and anti-TIMM44 (catalog HPA043052, Sigma) overnight at $4{ }^{\circ} \mathrm{C}$, and for $1.5 \mathrm{~h}$ at room temperature for secondary antibody incubation. After final washing, the nucleus visualized with DAPI (catalog C1005, Beyotime Institute of Biotechnology, Shanghai, China). The cells were observed using confocal microscopy.

\section{$\mathrm{NAD}^{+}$and NADH measurements}

Fresh mouse hippocampal brain tissue $(20 \mathrm{mg})$ was collected and homogenized in $400 \mu \mathrm{L} \mathrm{NAD}{ }^{+} / \mathrm{NADH}$ extract buffer on ice. Total $\mathrm{NAD}^{+}$and $\mathrm{NADH}$ were tested using an NAD $/ \mathrm{NADH}$ assay kit (Beyotime Institute of Biotechnology, Shanghai, China) according to the manufacturer's protocol [56]. NAD ${ }^{+} / \mathrm{NADH}$ ratio was calculated based on the valve of total $\mathrm{NAD}^{+}$and $\mathrm{NADH}\left(\mathrm{NAD}^{+}=\right.$total $\left.N A D^{+}-\mathrm{NADH}\right)$.

\section{Cell culture}

Hippocampal neuronal cells (HT22) and microglia cells (BV2) were respectively cultured in Dulbecco's modified Eagle's medium (DMEM), $10 \%$ fetal bovine serum (FBS) and $1 \%$ streptomycin and penicillin in incubator containing $5 \% \mathrm{CO}_{2}$ at $37^{\circ} \mathrm{C}$. To induce LPS-induced hippocampal neuron stress in vitro, BV2 cells were first exposed to LPS $(1 \mu \mathrm{g} / \mathrm{ml})$ for $24 \mathrm{~h}$, and the supernatants of LPS-stimulated microglia (Misup) were collected as previously described [57]. HT22 cells were then cultured in the Mi-sup for $24 \mathrm{~h}$.

\section{Co-immunoprecipitation (Co-IP) assays}

HT22 cell lysate was harvested, and Co-IP assay was performed using an Immunoprecipitation kit from Proteintech. Briefly, $2 \mu \mathrm{g}$ of antibodies against the proteins of interest or a negative control lgG of the same species were added to the cell lysate. Then the IP buffer was mixed with protein A/G beads. The immunoprecipitate was separated by SDS-PAGE and analyzed by western blotting.

\section{Plasmids and transfection}


The pDC315-SIRT3 was designed and purchased from Shanghai Gene-Pharma Co. (Shanghai, China). The plasmid was transfected into HEK293 cells and the supernatant was collected. The viral supernatant was identified and amplified to obtain adenovirus-SIRT3. The plasmids were transfected into the HT22 cells to overexpress SIRT3 with Lipofectamine 2000 (Thermo Fisher Scientific, Inc.) according to the manufacturer's protocol. Null vector transfection was used as the control group.

\section{ROS staining}

Total cellular ROS production was determined by detecting the fluorescence intensity of dichlorofluorescein diacetate (DCFH-DA) according to the manufacturer's protocol [58] (Reactive oxygen species assay kit, Beyotime, China). Briefly, HT22 cells were cultured in 12-well plates and incubated with freshly prepared DCFH-DA reagent in dark at $37^{\circ} \mathrm{C}$ for $30 \mathrm{~min}$. HT22 cells were washed three times with PBS and analyzed using fluorescence microscope.

\section{JC-1 staining}

JC-1 staining was used to evaluate MMP according to the manufacturer's protocol (Mitochondrial membrane potential assay kit with JC-1, Beyotime, China). Pretreated HT22 cells were washed three times with PBS, followed by the additional $1 \mathrm{ml} \mathrm{JC}-1$ working solution per sample and incubated at $37^{\circ} \mathrm{C}$ for 20 min. After incubation, HT22 cells were washed twice with JC-1 staining buffer and detected using fluorescence microscope.

\section{MPTP opening}

MPTP opening was measured by calcein-AM following the manufacturer's protocol (Mitochondrial permeability transition pore assay kit, Beyotime, China). HT22 cells were co-stained with $1 \mu \mathrm{M}$ calcein$\mathrm{AM}, 1 \mathrm{mM} \mathrm{CoCl}{ }_{2}$, and $200 \mathrm{nM}$ Mitotracker for 20 min at $37^{\circ} \mathrm{C}$. After staining, $\mathrm{HT} 22$ cells were washed with PBS and captured by fluorescent microscope. ImageJ software was used for quantification and measurement of fluorescent intensity.

\section{Western blot analysis}

Frozen hippocampus sample were homogenized in ice-cold extraction buffer. Homogenates were centrifuged at $12,000 \mathrm{~g}$ for $15 \mathrm{~min}$ at $4{ }^{\circ} \mathrm{C}$, the final supernatants were obtained. In contrast to frozen hippocampus tissues, HT22 cells were washed twice with PBS and lysed in an extraction buffer. Mitochondrial extracts were obtained with a mitochondrial isolation kit (Beyotime Institute of Biotechnology, Shanghai, China). Protein concentrations were determined by BCA Protein Assay Kit (Beyotime Institute of Biotechnology, Shanghai, China). Equal amounts of protein were separated on a $10 \%$ SDS-PAGE and transferred to a PVDF membrane. After being blocked, the membranes were incubated with correspondent primary antibody at $4{ }^{\circ} \mathrm{C}$ for overnight. Antibodies against a-tubulin (catalog 11244-1-AP), GAPDH (catalog 10494-1-AP), Bcl-2 (catalog 12789-1-AP), Bax (catalog 50599-2Ig), Cytochrome C (catalog 66264-1-lg), SHC (catalog 10054-1-AP) were obtained from Proteintech Group. 
Antibodies against VDAC (catalog D73D12), SIRT3 (catalog D22A3), PSD95 (catalog D27E11), BDNF (catalog 47808S) were from Cell Signaling Technology. Anti-Cyclophilin 40 (catalog 181983), anti-acetyl Lysine (catalog 80178) and anti-SHC phosphor S36 (catalog 54518) were from Abcam. Anti-JNK (catalog 7345) and anti-p-JNK (catalog 6254) were from Santa Cruz Biotechnology. After washing, the membrane was incubated with secondary antibodies (catalog SA00001-2, Proteintech Group) for 80 min at room temperature. Immunoreactive protein bands were detected using chemiluminescence reagents (Beyotime Institute of Biotechnology, Shanghai, China). In the present study, a-tubulin and GAPDH were used as loading control in hippocampus homogenates and whole-cell extracts, while VDAC was used as loading control in both hippocampus and cell mitochondrial extracts.

\section{Statistical analysis}

Data were analyzed using GraphPad Prism 8 software and the data were presented as mean \pm standard error. Statistical significance was evaluated by the Student's $t$-test for comparing two experimental groups. For datasets having multiple groups, statistical significance was evaluated using a one-way analysis of variance (ANOVA) followed by Tukey's test for the post hoc analysis. Results were considered as significant at $P<0.05$.

\section{Results}

\section{SIRT3 expression is diminished in LPS-induced mice hippocampus}

We first determined whether mitochondrial sirtuins (SIRT3, SIRT4 and SIRT5) expression levels were altered in the hippocampus of LPS-treated mice. The mRNA level of SIRT3 was significantly decreased 24 h after $10 \mathrm{mg} / \mathrm{kg}$ LPS i.p. (Fig. 1A). In addition, we performed analysis on existing GEO database of peripheral blood mononuclear cell (PBMC) from healthy or septic patients (GSE28750 and GSE95233), revealing a down-regulation of SIRT3 gene expression (Fig. 1B). Western blot analysis confirmed that SIRT3 was significantly reduced in both whole-hippocampal homogenates and mitochondrial fractions in mice $24 \mathrm{~h}$ after $10 \mathrm{mg} / \mathrm{kg}$ LPS i.p. (Fig. 1C and 1D). Consistent with the western blot analysis, the immunofluorescence staining confirmed that the expression of SIRT3 was significantly decreased (Fig. 1E).

\section{Sirt3 Deficiency Exacerbates Lps-induced Hippocampus-dependent Cognitive Impairments}

The severity of cognitive impairments was evaluated in LPS-treated WT and SIRT3-/-mice. Mice received open field test (OFT) on day 5 and fear conditioning test (FCT) on day 6 and 7 after LPS exposure (Fig. 2A). Compared with WT Con mice, SIRT3-/-mice resulted in decreased survival rate within $7 \mathrm{~d}$ after LPS exposure (Fig. 2B). Compared with WT LPS mice, SIRT3-/-mice resulted in declined contextual freezing time after LPS exposure (Fig. 2C). Compared with WT Con mice, both WT LPS and SIRT3-/-mice contributed to the decreasing of post-tone freezing time in the auditor-cued fear conditioning test, 
however, there was no difference in the freezing time between two SIRT3-/-mice groups (Fig. 2D). The OFT was performed to determine whether the locomotor activity of mice was altered. There was no difference between four groups for the total distance (Fig. 2E) and the time spent in the center of arena (Fig. 2F). The results indicated that SIRT3 deficiency exacerbates LPS-induced hippocampus-dependent cognitive impairments.

\section{SIRT3 deficiency exacerbates hippocampal neuronal apoptosis and synaptic dysfunction in LPS-induced SAE}

We used Nissl's staining to assess whether SIRT3 deficiency lead to hippocampal neuron stress after LPS exposure. In dentate gyrus (DG) region of the hippocampus, WT Con group hardly had any neuronal damage, while the decreasing numbers of surviving neurons and irregular arrangement neurons were observed in the WT LPS group. In comparison with WT LPS group, remarkably increased numbers of Nissl-positive neurons and significantly decreased thickness of DG region granule cell layer were detected in SIRT3-/-LPS group, which indicating exacerbated hippocampal neuronal damage (Fig. 3A and 3C).

Then, TUNEL staining was utilized to explore the hippocampal apoptosis cells at $24 \mathrm{~h}$ post LPS induction. The numbers of TUNEL-positive cells in DG region were significantly increased in SIRT3-/-LPS group compared to WT LPS group. While both WT Con and SIRT3-/-Con group hardly observed TUNEL-positive cells (Fig. 3B and 3D).

Synaptic dysfunction and degeneration contribute to the deterioration of memory performance $[28,29]$. Synapse-associated proteins (postsynaptic density protein 95, PSD95; brain derived neurotrophic factor precursor, pro-BDNF) were assessed by western blot in hippocampus (Fig. 3E). PSD 95 levels were remarkably declined in WT LPS group in comparison with WT Con group, and showed further decline in SIRT3-/-LPS mice. Pro-BDNF levels were significantly increased in SIRT3 deficiency mice in comparison with WT mice, however, the levels within WT and SIRT3-/-groups had no significant differences. All these results indicated that SIRT3 deficiency exacerbated hippocampal neuron apoptosis and synaptic dysfunction in LPS-induced SAE in mice.

\section{SIRT3 deficiency decreased the hippocampal NAD + /NADH ratio and accelerated mitochondrial apoptosis in LPS-induced SAE}

Mitochondrial lysine acetylation level was increased in WT LPS mice compared with WT Con mice, and was further increased in SIRT3-/-LPS mice (Fig. 4A). Since SIRT3 is NAD -dependent, we tested the effect of LPS exposure on NAD $/ \mathrm{NADH}$ ratio in fresh hippocampus. The $\mathrm{NAD}^{+}$content was significantly declined in WT LPS mice compared with WT Con mice, and was further declined in SIRT3-/-LPS mice (Fig. 4B). Similar trends of $\mathrm{NAD}^{+} / \mathrm{NADH}$ ratio were also witnessed in four groups (Fig. 4C). These results indicated that hippocampal mitochondrial function was damaged after LPS treatment, and this damage was further exacerbated in SIRT3-/-mice. 
Mitochondrial apoptosis-associated protein B-cell lymphoma 2 (Bcl-2), Bax and cytochrome C (Cyt C) in mitochondrial and cytosol extracts of hippocampus were accessed by western blot respectively. VDAC, the distinctive protein of mitochondrial, was used as normalization for mitochondrial extracts. Compared with WT LPS mice, the ratio of Bcl-2/Bax in mitochondrial extracts was sharply decreased in SIRT3-/-LPS mice, while the ratio in cytosol was increased. Similar trends were showed on the change of Cyt $C$ in these two groups (Fig. 4D and 4E). These results showed that increased $\mathrm{Bcl}-2$ and cytochrome $\mathrm{C}$ were released to cytosol from mitochondrial after LPS exposure in SIRT3-/-mice, indicating accelerated mitochondrial apoptosis in LPS-induced neurological impairment.

\section{SIRT3-mediated deacetylation of CypD contributed to increased ROS generation, declined MMP and increased MPTP opening in HT22 cells with LPS-induced microglia supernatants}

Subsequently, we determined how SIRT3 deficiency promoted mitochondrial dysfunction in hippocampal neurons. Immunofluorescent staining revealed co-localization of SIRT3 and NeuN in hippocampus

(Fig. 5A). HT22 cells (a hippocampal neuronal cell line) were then taken for experiments.

To mimic LPS-induced hippocampal neuron stess in vitro, BV2 cells (a microglia-derived cell line) were first exposed to LPS $(1 \mu \mathrm{g} / \mathrm{ml})$ for $24 \mathrm{~h}$, and the supernatants of LPS-stimulated microglia (Mi-sup) were collected. HT22 cells were then cultured in the Mi-sup for $24 \mathrm{~h}$. In HT22 cells, SIRT3 levels were decreased with Mi-sup rather than LPS $(1 \mu \mathrm{g} / \mathrm{ml})$ treatment. Mi-sup treatment downregulated PSD95 and upregulated pro-BDNF levels (Fig. 5B). Therefore, LPS did not directly alter PSD 95 and pro-BDNF levels, but the Mi-sup attributed to synaptic dysfunction in HT22 cells. Mi-sup were then used in the following experiments.

Cyclophilin D (CypD) is the key component of mitochondrial MPTP and lysine acetylation (Ace-K) levels of CypD leads to an increased susceptibility to MPTP opening [30,31]. Compared with control group, CypD Ace-K levels were significantly increased after Mi-sup exposure (Fig. 5C). Next, CypD Ace-K levels were evaluated in HT22 cells with different SIRT3 deacetylation activity, by means of pretreated with 50 $\mu \mathrm{M}$ 3-TYP (SIRT3 deacetylation activity inhibitor) or transfected with SIRT3 overexpression plasmid (SIRT3-oe). CypD Ace-K levels were increased pretreated with 3-TYP after Mi-sup exposure, while decreased with SIRT3 overexpression, indicating that CypD Ace-K levels were significantly altered with different SIRT3 deacetylation activity in HT22 after Mi-sup treatment (Fig. 5D and 5E).

Compared with control group, ROS generation were increased with Mi-sup treatment, the effects of which were further increased with 3-TYP pretreatment. After SIRT3 overexpression, the effects of ROS generation were significantly inhibited (Figure 5F). MPTP opening and MMP were evaluated by observing the relative fluorescence intensity of calcein $\mathrm{AM}, \mathrm{Co}^{2+}$ and $\mathrm{JC}-1$ in four groups (Fig. $5 \mathrm{G}$ and $5 \mathrm{H}$ ). MPTP opening was increased in HT22 cells after Mi-sup treatment, while it further increased in Mi-sup+3-TYP group, and the effects were attenuated in Mi-sup+SIRT3-oe group. These results indicated that SIRT3mediated deacetylation of CypD contributed to increased ROS generation, declined MMP and MPTP opening in HT22 cells with LPS-induced microglia supernatants. 


\section{SIRT3 deacetylation activity contributed to synaptic dysfunction and mitochondrial apoptosis in HT22 cells with LPS-induced microglia supernatant via JNK/p66Shc pathway}

Several pathways capable of regulating ROS production and mitochondrial apoptosis, among which the phosphorylation of serine (Ser) 36 in p66Shc has been indicated as a key regulatory step. P66Shc translocate to the mitochondria and oxidizes Cyt $\mathrm{C}$ to yield $\mathrm{H}_{2} \mathrm{O}_{2}$, which in turn initiates cell apoptosis. Studies indicated that p-p66Shc-Ser36 was regulated by JNK [32]. Meanwhile, JNK1/2 can translocate to the mitochondrial under stress resulting in accelerated p66Shc phosphorylation, which in turn increased ROS production and cell death $[33,34]$. But it remains unclear whether SIRT3 deacetylation activity was involved in this key process.

The levels of p-p66Shc-Ser36 and p-JNK in hippocampal mitochondrial extracts were remarkably increased in WT LPS mice in comparison with WT Con mice, and both levels were further increased in SIRT3-/-LPS mice, indicating that SIRT3 deficiency exacerbates LPS-induced synaptic dysfunction via the activation of mitochondrial JNK and p66Shc (Fig. 6A).

In HT22 cells, Mi-sup treatment significantly downregulated PSD95 and upregulated pro-BDNF levels, and these changes were partly abolished by SIRT3 overexpression. The ratio of Bcl-2/Bax and Cyt C levels were decreased after Mi-sup treatment, and these phenomena were partly reversed by SIRT3 overexpression (Fig. 6B). The ratio of p-p66Shc-Ser36/p66Shc and p-JNK/JNK in HT22 mitochondrial extracts were increased after Mi-sup treatment. After SIRT3 overexpression, the mitochondrial p-p66ShcSer36 and p-JNK levels were sharply decreased after Mi-sup treatment. These results indicated that SIRT3 can protect HT22 cells from mitochondrial apoptosis after Mi-sup treatment by regulating mitochondrial JNK and p66Shc activation (Fig. 6C).

In HT22 cells, the changes of PSD95 and pro-BDNF levels after Mi-sup treatment were further exacerbated in Mi-sup+3-TYP group, and the levels could be reversed by $30 \mu \mathrm{M}$ SP600125 (JNK inhibitor) pretreatment. The ratio of $\mathrm{Bcl}-2 / \mathrm{Bax}$ and $\mathrm{Cyt} \mathrm{C}$ levels were further decreased in Mi-sup+3-TYP group, which were reversed by SP600125 pretreatment (Fig. 6D). The increasing of p-p66Shc-Ser36/p66Shc and p-JNK/JNK in HT22 mitochondrial extracts were further exacerbated in Mi-sup+3-TYP group. Then, we witnessed the level of p-p66Shc-Ser36 was inhibited by SP600125 pretreatment, which indicated that p66Shc-Ser36 phosphorylation was regulated by JNK activation after Mi-sup exposure (Fig. 6E). To corroborate these results, mitochondrial p-p66Shc-Ser36 accumulation was determined by immunofluorescence (Fig. 6F). In agreement with western blotting results, p-p66Shc-Ser36 co-localized with TIMM44 in HT22 cells was witnessed after Mi-sup treatment, and the phenomenon of co-localization of p-p66Shc-Ser36 with mitochondrial was further increased in Mi-sup+3-TYP group, which was decreased after SIRT3 overexpression. These data indicated that JNK/p66Shc pathway was involved in SIRT3 deacetylation activity mediated mitochondrial apoptosis in HT22 cells with LPS-induced microglia supernatants.

\section{Discussion}


Our study showed that SIRT3 deficiency exacerbates sepsis-induced hippocampus-dependent cognitive impairments via enhancing oxidative stress and synaptic dysfunction in mice. Our study showed that SIRT3-mediated CypD deacetylation was involved in LPS-induced hippocampal synaptic dysfunction, via ROS accumulation, declined MMP, increased MPTP opening, mitochondrial Cyt $C$ release and mitochondrial apoptosis of hippocampal neuron via JNK/p66Shc pathway. Our results revealed that SIRT3 may be a promising therapeutic and diagnostic target for cognitive dysfunction induced by SAE.

WT mice treated with $10 \mathrm{mg} / \mathrm{kg}$ LPS in our study showed declined freezing time in both contextual and cue memory, suggesting that our SAE model was successful. Compared with SIRT3-/-Con group, the mice in SIRT3-/-LPS group showed significantly declined hippocampal-dependent cognitive dysfunction, indicating that hippocampus was more susceptible to be affected by LPS exposure.

SIRT3 has been reported to regulate almost every aspect of mitochondrial function like mitochondrial biogenesis and dynamics [35, 36, 37, 438], ROS metabolism, ATP production and maintenance of mitochondrial integrity $[39,40]$. It was expressed maximally in metabolically active tissues like brain, heart, kidney, liver, brown adipose tissue and skeletal muscles. Altered mitochondrial function in the brain, like declined ATP production and increased oxidative damage from ROS, was witnessed in different SIRT3 deacetylation activity under different stress [41, 42]. SIRT3 deficiency mice showed mild hippocampal-dependent cognitive dysfunction and morphologic change of neurons in DG region, but significantly exacerbated after LPS treatment in our study. This phenomenon indicated that SIRT3 knockout mice exhibited almost normal physiology under normal condition, but tended to develop deteriorated brain dysfunction and poor remote memory under stress $[43,44]$.

At the cellular level, previous studies found that HT22 cells responded to Mi-sup rather than LPS in cell viability and neuron apoptosis. LPS did not increase $\mathrm{Ca}^{2+}$ and neither promoted neuronal apoptosis in cultured primary hippocampal neurons expressing low levels of TLR4 [45]. LPS may not directly impair neurons but induce neuroinflammation by firstly activating microglia. Therefore, LPS-stimulated microglia supernatant was used for the condition medium in our study. We witnessed increased CypD Ace-K levels, increased ROS generation, decreased MMP and increased MPTP opening and declined protective synaptic protein levels in HT22 cells after Mi-sup exposure, which further deteriorated with 3TYP pretreatment. ROS production and low MMP are two inducers to MPTP opening. A long-lasting MPTP opening can lead to irreversible consequesces, including matrix swelling, dissipation of mitochondrial potential, ATP hydrolysis into ADP, and uncoupling of oxidative phosphorylation, causing cell death [46]. These results indicated that hippocampal neurons with decreased SIRT3 deacetylation activity were prone to suffer from mitochondrial apoptosis and synaptic dysfunction after Mi-sup exposure. However, the extracellular microenvironment and the specific interaction between hippocampal neuron and microglia remain to be explored.

Our study found that LPS exposure activated JNK and induced mitochondrial p66Shc-Ser36 phosphorylation. P66Shc plays an important role in the generation of mitochondrial ROS and serves as antioxidants in many pathological conditions and disease $[47,48]$. PKC $\beta$ has been proposed as p66Shc- 
Ser36 kinase but JNKs may also phosphorylate this residue. Studies showed application of specific inhibitors caused a pronounced decrease in p66Shc-Ser36 phosphorylation only in the case of JNK1/2, and p-p66Shc-Ser36-dependent ROS production and mitochondrial apoptosis was sharply diminished in JNK1/2 deficient MEFs [49], indicating JNK was critical for p66Shc activation. In our study, we showed that phosphorylation of p66Shc-Ser36 and JNK were regulated by SIRT3 deacetylation activity, and phosphorylation of p66Shc-Ser36 was regulated by JNK activation. These data indicated that SIRT3mediated JNK/p66Shc pathway was involved in mitochondrial apoptosis and synaptic dysfunction in HT22 cells with LPS-induced microglia supernatants.

\section{Conclusions}

In summary, the present study suggested that SIRT3-mediated CypD deacetylation was involved in LPSinduced hippocampal synaptic dysfunction, via ROS accumulation, declined MMP, increased MPTP opening, mitochondrial Cyt $C$ release and mitochondrial apoptosis of hippocampal neuron via JNK/p66Shc pathway. Our results revealed that SIRT3 may be a promising therapeutic and diagnostic target for cognitive dysfunction induced by SAE.

\section{Abbreviations}

SAE: Sepsis-associated encephalopathy; NAD+: Nicotinamide adenine dinucleotide; LPS:

Lipopolysaccharide; CypD: Cyclophilin D; ROS: Reactive oxygen species; MMP: Mitochondrial membrane potential; MPTP: Membrane permeability transition pore; Bcl-2: B-cell lymphoma 2; Cyt C: Cytochrome C; OFT: Open field test; FCT: Fear conditioning test; TUNEL: TdT-mediated dUTP Nick-End Labeling; DG: Dentate gyrus

\section{Declarations}

\section{Ethics approval and consent to participate}

Procedures involving animals were approved by the Animal Experimentation Ethics Committee of Ruijin Hospital, and were performed strictly according to the National Institutes of Health Guidelines for the Care and Use of Laboratory Animals.

\section{Consent for publication}

Not applicable

\section{Availability of data and materials}

The data and materials of this study are available from the corresponding authors for reasonable requests.

\section{Competing interests}


The authors declare that they have no competing interests.

\section{Funding}

This work was supported by the National Natural Science Foundation of China [grant number 81871101] and Shanghai Sailing Program [grant number 19YF1430600]

\section{Author's contributions}

ZC.L., JB.L. and Y.L. conceived and designed the study; ZC.L. and SY.L. performed the experiments and analyzed data with assistance from YJ.L., LF.Y., F.C., Q.T. and Y.Y.; ZC.L., JB.L. and Y.L. coordinated the project, interpreted results, and wrote the paper; JB.L. and Y.L. supervised the project.

\section{Acknowledgements}

Not applicable

\section{References}

1. Rhodes A, Evans LE, Alhazzani W, Levy MM, Antonelli M, Ferrer R, et al. Surviving sepsis campaign: International Guidelines for Management of Sepsis and Septic Shock: 2016. Intensive Care Med. 2017; 43: 304-77.

2. Chung HY, Wickel J, Brunkhorst FM \& Geis C. Sepsis-Associated Encephalopathy: From Delirium to Dementia? J Clin Med. 2020; 9: 703.

3. Mazeraud A, Righy C, Bouchereau E, Benghanem S, Bozza FA \& Sharshar T. Septic-Associated Encephalopathy: A Comprehensive Review. Neurotherapeutics. 2020; 17: 392-403.

4. Haileselassie B, Joshi AU, Minhas PS, Mukherjee R, Andreasson KI \& Mochly-Rosen D. Mitochondrial dysfunction mediated through dynamin-related protein 1 (Drp1) propagates impairment in blood brain barrier in septic encephalopathy. J Neuroinflammation. 2020; 17: 36.

5. Kikuchi DS, Campos ACP, Qu H, Forrester SJ, Pagano RL, Lassègue B, et al. Poldip2 mediates bloodbrain barrier disruption in a model of sepsis-associated encephalopathy. J Neuroinflammation. 2019; 16: 241.

6. Tauber SC, Djukic M, Gossner J, Eiffert H, Brück W \& Nau R. Sepsis-associated encephalopathy and septic encephalitis: an update. Expert Rev Anti Infect Ther. 2021; 19: 215-31.

7. Wu J, Zhang M, Hao S, Jia M, Ji M, Qiu L, et al. Mitochondria-Targeted Peptide Reverses Mitochondrial Dysfunction and Cognitive Deficits in Sepsis-Associated Encephalopathy. Mol Neurobiol. 2015; 52: 783-91.

8. Xie K, Wang Y, Yin L, Wang Y, Chen H, Mao X, et al. Hydrogen Gas Alleviates Sepsis-Induced Brain Injury by Improving Mitochondrial Biogenesis Through the Activation of PGC-a in Mice. Shock. 2021; 55: 100-9. 
9. Shadel Gerald S \& Horvath Tamas L. Mitochondrial ROS signaling in organismal homeostasis. Cell. 2015; 163: 560-9.

10. Willems PH, Rossignol R, Dieteren CE, Murphy MP \& Koopman WJ. Redox Homeostasis and Mitochondrial Dynamics. Cell Metab. 2015; 22: 207-18.

11. Pemberton JM, Pogmore JP \& Andrews DW. Neuronal cell life, death, and axonal degeneration as regulated by the BCL-2 family proteins. Cell Death Differ. $2021 ; 28: 108-22$.

12. Xian H, Liu Y, Rundberg Nilsson A, Gatchalian R, Crother TR, Tourtellotte WG, et al. Metformin inhibition of mitochondrial ATP and DNA synthesis abrogates NLRP3 inflammasome activation and pulmonary inflammation. Immunity. 2021; 54: 1463-77.

13. Chang X, Zhang T, Liu D, Meng Q, Yan P, Luo D, et al. Puerarin Attenuates LPS-Induced Inflammatory Responses and Oxidative Stress Injury in Human Umbilical Vein Endothelial Cells through Mitochondrial Quality Control. Oxid Med Cell Longev. 2021; 2021: 6659240.

14. Kurundkar D, Kurundkar AR, Bone NB, Becker EJ Jr, Liu W, Chacko B, et al. SIRT3 diminishes inflammation and mitigates endotoxin-induced acute lung injury. JCl Insight. 2019; 4: e120722.

15. Xu Y, Zhang S, Rong J, Lin Y, Du L, Wang Y, et al. Sirt3 is a novel target to treat sepsis induced myocardial dysfunction by acetylated modulation of critical enzymes within cardiac tricarboxylic acid cycle. Pharmacol Res. 2020; 159: 104887.

16. Xin T \& Lu C. SirT3 activates AMPK-related mitochondrial biogenesis and ameliorates sepsis-induced myocardial injury. Aging (Albany NY). 2020; 12: 16224-37.

17. Zhao W, Zhang L, Chen R, Lu H, Sui M, Zhu Y, et al. SIRT3 Protects Against Acute Kidney Injury via AMPK/mTOR-Regulated Autophagy. Front Physiol. 2018; 9: 1526.

18. Tan C, Gu J, Li T, Chen H, Liu K, Liu M, et al. Inhibition of aerobic glycolysis alleviates sepsisinduced acute kidney injury by promoting lactate/Sirtuin 3/AMPKregulated autophagy. Int J Mol Med. 2021; 47: 19.

19. Kent AC, El Baradie KBY \& Hamrick MW. Targeting the Mitochondrial Permeability Transition Pore to Prevent Age-Associated Cell Damage and Neurodegeneration. Oxid Med Cell Longev. 2021; 2021: 6626484.

20. Gordan R, Fefelova N, Gwathmey JK \& Xie LH. Iron Overload, Oxidative Stress and Calcium Mishandling in Cardiomyocytes: Role of the Mitochondrial Permeability Transition Pore. Antioxidants (Basel). 2020; 9: 758.

21. Castillo EC, Morales JA, Chapoy-Villanueva H, Silva-Platas C, Treviño-Saldaña N, Guerrero-Beltrán CE, et al. Mitochondrial Hyperacetylation in the Failing Hearts of Obese Patients Mediated Partly by a Reduction in SIRT3: The Involvement of the Mitochondrial Permeability Transition Pore. Cell Physiol Biochem. 2019; 53: 465-79.

22. Katwal G, Baral D, Fan X, Weiyang H, Zhang X, Ling L, et al. SIRT3 a Major Player in Attenuation of Hepatic Ischemia-Reperfusion Injury by Reducing ROS via Its Downstream Mediators: SOD2, CYP-D, and HIF-1a. Oxid Med Cell Longev. 2018; 2018: 2976957. 
23. Wu J, Deng Z, Sun M, Zhang W, Yang Y, Zeng Z, et al. Polydatin protects against lipopolysaccharideinduced endothelial barrier disruption via SIRT3 activation. Lab Invest. 2020; 100: 643-56.

24. Ray PD, Huang BW \& Tsuji Y. Reactive oxygen species (ROS) homeostasis and redox regulation in cellular signaling. Cell Signal. 2012; 24: 981-90.

25. Miyazawa M \& Tsuji Y. Evidence for a novel antioxidant function and isoform-specific regulation of the human p66Shc gene. Mol Biol Cell. 2014; 25: 2116-27.

26. Mir HA, Ali R, Mushtaq U \& Khanday FA. Structure-functional implications of longevity protein p66Shc in health and disease. Ageing Res Rev. 2020; 63: 101139.

27. Kumar S, Kim YR, Vikram A, Naqvi A, Li Q, Kassan M, et al. Sirtuin1-regulated lysine acetylation of p66Shc foverns diabetes-induced vascular oxidative stress and endothelial dysfunction. PNAS. 2017; 114: 1714-9.

28. Cisternas P, Salazar P, Serrano FG, Montecinos-Oliva C, Arredondo SB, Varela-Nallar L, et al. Fructose consumption reduces hippocampal synaptic plasticity underlying cognitive performance. Biochim Biophys Acta. 2015; 1852: 2379-90.

29. Doost Mohammadpour J, Hosseinmardi N, Janahmadi M, Fathollahi Y, Motamedi F \& Rohampour K. Non-selective NSAID Improve the amyloid-beta-mediated suppression of memory and synaptic plasticity. Pharmacol Biochem Behav. 2015; 132: 33-41.

30. Wang NN, Xu HH, Zhou W, Yang HX, Wang J, Ma ZC, et al. Aconitine attenuates mitochondrial dysfunction of cardiomyocytes via promoting deacetylation of cyclophilin-D mediated by sirtuin-3. J Ethnopharmacol. 2021; 270: 113765.

31. Castillo EC, Morales JA, Chapoy-Villanueva H, Silva-Platas C, Treviño-Saldaña N, Guerrero-Beltrán CE, et al. Mitochondrial Hyperacetylation in the Failing Hearts of Obese Patients Mediated Partly by a Reduction in SIRT3: The Involvement of the Mitochondrial Permeability Transition Pore. Cell Physiol Biochem. 2019; 53: 465-79.

32. Haller M, Khalid S, Kremser L, Fresser F, Furlan T, Hermann M, et al. Novel Insights into the PKC $\beta$ dependent Regulation of the Oxidoreductase p66Shc. J Biol Chem. 2016; 291: 23557-68.

33. Chambers JW \& LoGrasso PV. Mitochondrial c-Jun N-terminal kinase (JNK) signaling initiates physiological changes resulting in amplification of reactive oxygen species generation. J Biol Chem. 2011; 286: 16052-62.

34. Chambers JW, Pachori A, Howard S, Iqbal S \& LoGrasso PV. Inhibition of JNK mitochondrial localization and signaling is protective against ischemia/reperfusion injury in rats. J Biol Chem. 2013; 288: 4000-11.

35. Hasan-Olive MM, Lauritzen KH, Ali M, Rasmussen LJ, Storm-Mathisen J \& Bergersen LH. A Ketogenic Diet Improves Mitochondrial Biogenesis and Bioenergetics via the PGC1a-SIRT3-UCP2 Axis. Neurochem Res. 2019; 44: 22-37.

36. Liu H, Li S, Liu X, Chen Y \& Deng H. SIRT3 Overexpression Inhibits Growth of Kidney Tumor Cells and Enhances Mitochondrial Biogenesis. J Proteome Res. 2018; 17: 3143-52. 
37. Klimova N, Long A \& Kristian T. Nicotinamide mononucleotide alters mitochondrial dynamics by SIRT3-dependent mechanism in male mice. J Neurosci Res. 2019; 97: 975-90.

38. Liu J, Yan W, Zhao X, Jia Q, Wang J, Zhang H, et al. Sirt3 attenuates post-infarction cardiac injury via inhibiting mitochondrial fission and normalization of AMPK-Drp1 pathways. Cell Signal. 2019; 53: 113.

39. Shen Y, Wu Q, Shi J \& Zhou S. Regulation of SIRT3 on mitochondrial functions and oxidative stress in Parkinson's disease. Biomed Pharmacother. 2020; 132: 110928.

40. Bause AS \& Haigis MC. SIRT3 regulation of mitochondrial oxidative stress. Exp. Gerontol. 2013; 48: 634-9.

41. Liu Q, Sun YM, Huang H, Chen C, Wan J, Ma LH, et al. Sirtuin 3 protects against anesthesia/surgeryinduced cognitive decline in aged mice by suppressing hippocampal neuroinflammation. $\mathrm{J}$ Neuroinflammation. 2021; 18: 41.

42. Liu Y, Cheng A, Li YJ, Yang Y, Kishimoto Y, Zhang S, et al. SIRT3 mediates hippocampal synaptic adaptations to intermittent fasting and ameliorates deficits in APP mutant mice. Nat Commun. 2019; 10: 1886.

43. Lombard DB \& Zwaans BM. SIRT3: as simple as it seems? Geronto 60, 56-64 (2014).

44. Kim H, Kim S, Choi JE, Han D, Koh SM, Kim HS, et al. Decreased neuron number and synaptic plasticity in SIRT3 knockout mice with poor remote memory. Neurochem Res. 2019; 44: 676-82.

45. Calvo-Rodríguez M, De IFC, García-Durillo M, García-Rodríguez C, Villalobos C \& Núñez L. Aging and amyloid $\beta$ oligomers enhance TLR4 expression, LPS-induced $\mathrm{Ca}(2+)$ responses, and neuron cell death in cultured rat hippocampal neurons. J Neuroinflammation. 2017; 14: 24.

46. Morciano G, Naumova N, Koprowski P, Valente S, Sardão VA, Potes Y, et al. The mitochondrial permeability transition pore: an evolving concept critical for cell life and death. Biol Rev Camb Philos Soc (2021).

47. Cosentino F, Francia P, Camici GG, Pelicci PG, Lüscher TF \& Volpe M. Final common molecular pathways of aging and cardiovascular disease: role of the p66Shc protein. Arterioscler Thromb Vasc Biol. 2008; 28: 622-8.

48. Galimov ER. The role of p66shc in oxidative stress and apoptosis. Acta Naturae. 2010; 2: 44-51.

49. Khalid S, Drasche A, Thurner M, Hermann M, Ashraf MI, Fresser F, et al. cJun N-terminal kinase (JNK) phosphorylation of serine 36 is critical for p66Shc activation. Sci Rep. 2016; 6: 20930.

50. Hoogland IC, Houbolt C, van Westerloo DJ, van Gool WA \& van de Beek D. Systemic inflammation and microglial activation: systematic review of animal experiments. J Neuroinflammation. 2015; 12: 114.

51. Leite HR, Oliveira-Lima OC, Pereira LM, Oliveira VEM, Prado VF, Prado MAM, et al. Vesicular acetylcholine transporter knock down-mice are more susceptible to inflammation, c-Fos expression and sickness behavior induced by lipopolysaccharide. Brain Behav Immun. 2016; 57: 282-92. 
52. Bhaskar K, Konerth M, Kokiko-Cochran ON, Cardona A, Ransohoff RM \& Lamb BT. Regulation of tau pathology by the microglial fractalkine receptor. Neuron. 2010; 68: 19-31.

53. Liu Q, Sun YM, Huang H, Chen C, Wan J, Ma LH, et al. Sirtuin 3 protects against anesthesia/surgeryinduced cognitive decline in aged mice by suppressing hippocampal neuroinflammation. $J$ Neuroinflammation. 2021; 18: 41.

54. Rocha M, Wang D, Avila-Quintero V, Bloch MH \& Kaffman A. Deficits in hippocampal-dependent memory across different rodent models of early life stress: systematic review and meta-analysis. Transl Psychiatry. 2021; 11: 231.

55. Xie J, Zhao ZZ, Li P, Zhu CL, Guo Y, Wang J, et al. Senkyunolide I Protects against Sepsis-Associated Encephalopathy by Attenuating Sleep Deprivation in a Murine Model of Cecal Ligation and Puncture. Oxid Med Cell Longev. 2021; 2021: 6647258.

56. Yin J, Nielsen M, Li S \& Shi J. Ketones improves Apolipoprotein E4-related memory deficiency via sirtuin 3. Aging (Albany NY). 2019; 11: 4579-86.

57. Liu Y, Zhang Y, Zheng X, Fang T, Yang X, Luo X, et al. Galantamine improves cognition, hippocampal inflammation, and synaptic plasticity impairments induced by lipopolysaccharide in mice. $J$ Neuroinflammation. 2018; 15: 112.

58. Zhao Y, Tang J, Yang D, Tang C \& Chen J. Staphylococcal enterotoxin M induced inflammation and impairment of bovine mammary epithelial cells. J Dairy Sci. 2020; 103: 8350-9.

\section{Figures}


A

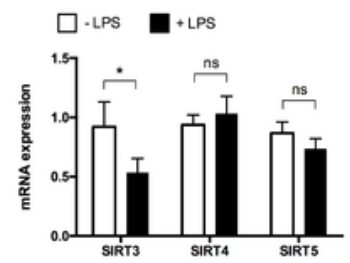

C

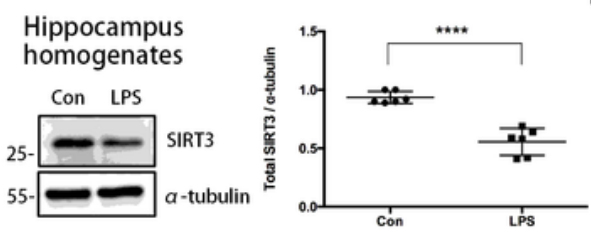

Hippocampus

\begin{tabular}{l} 
Con LPS \\
a-tubulin \\
\hline$-\infty$
\end{tabular}

E
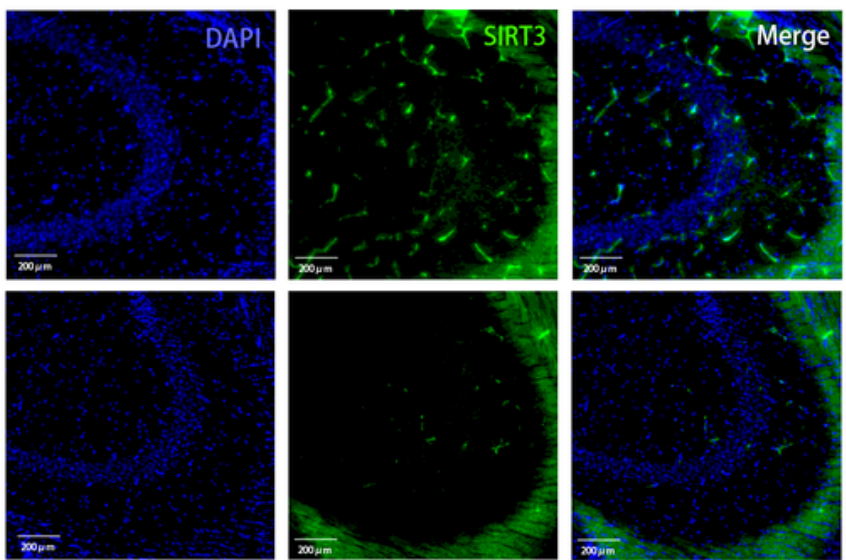

LPS

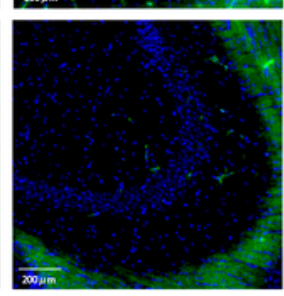

B
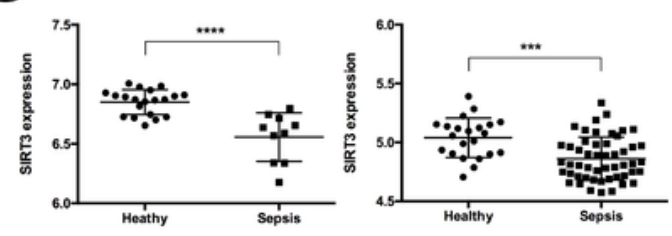

D
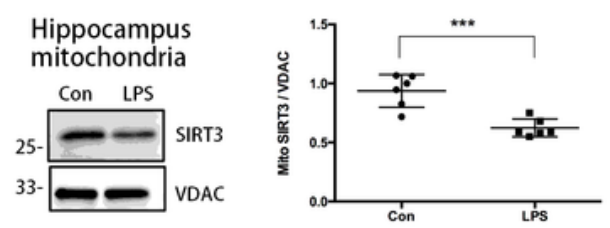

\section{Figure 1}

SIRT3 expression is diminished in LPS-induced mice hippocampus. Mice were treated with $10 \mathrm{mg} / \mathrm{kg}$ LPS i.p. and euthanized after $24 \mathrm{~h}$. (A) Detection of hippocampal SIRT3, SIRT4 and SIRT 5 mRNA abundances using RT-PCR. The mRNA level was normalized to the mRNA level of GAPDH as an endogenous control. Bars indicate the means \pm SD; $P$ values were determined using a t-test: ${ }^{*} P<0.05$. (B) The difference of PBMC SIRT3 gene expression between healthy control and septic patients was analyzed using GSE 
database. In GSE28750 database, healthy group: $n=20$, sepsis group: $n=10$. In GSE95233 database, healthy group: $n=22$, sepsis group: $n=51$. $P$ values were determined using a t-test: ${ }^{*} * 2<0.001$; $\star \star \star \star x<0.0001$. The protein levels of hippocampal homogenates $(C)$ and mitochondrial fractions (D) were examined by western blots. SIRT3 level was normalized to a-tubulin and VDAC as endogenous control.

Each symbol represents one mouse $(n=6)$. Bars indicate the means $\pm S D ; P$ values were determined using a t-test: ${ }^{\star *} P<0.001 ; * \star \star * P<0.0001$. (E) The immunofluorescence staining was performed with SIRT3 (green) and DAPI (blue) in hippocampus.

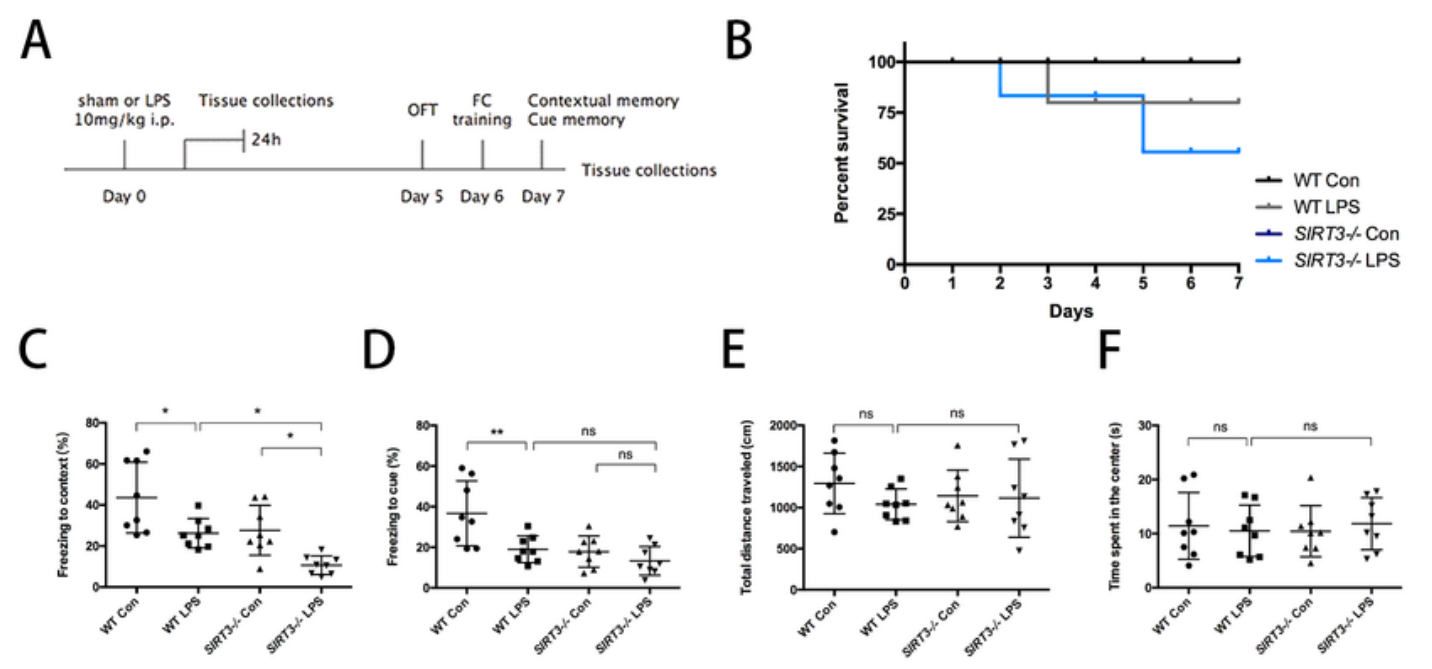




\section{Figure 2}

SIRT3 deficiency exacerbates LPS-induced hippocampus-dependent cognitive impairments. Mice were divided into 4 groups: WT Con, WT LPS, SIRT3-/-Con and SIRT3-/- LPS. (A) Schematic diagram of the experimental procedure. Mice received OFC on day 5 and FCT on day 6 and 7 after LPS exposure. Hippocampal tissues were collected on $24 \mathrm{~h}$ after $10 \mathrm{mg} / \mathrm{kg}$ LPS exposure. (B) The survival rate of the mice within $7 \mathrm{~d}$ after LPS exposure. Contextual memory test (C) and cue memory test (D) were assessed. Each symbol represents one mouse $(n=8)$. Bars indicate the means $\pm S D ; P$ values were determined using ANOVA and Tukey's post hoc analysis: ns, not significant; ${ }^{*} P<0.05 ;{ }^{* \star} P<0.01$. The ambulatory distance (E) and time spent in the center $(\mathrm{F})$ were detected in OFT. Each symbol represents one mouse $(n=8)$. Bars indicate the means $\pm S D ; P$ values were determined using ANOVA and Tukey's test for the post hoc analysis: ns, not significant. 
A

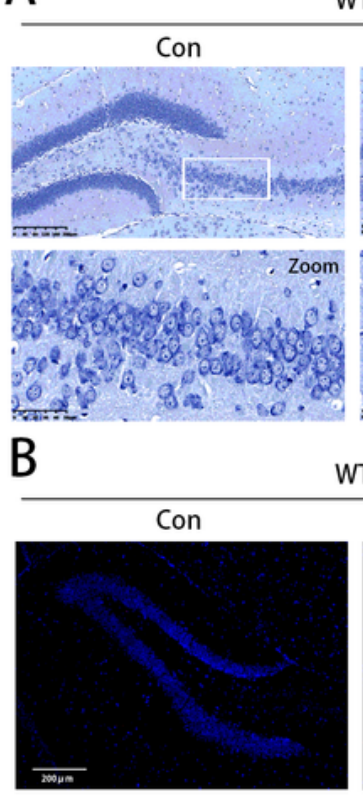

C

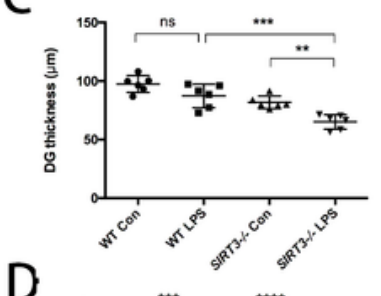

D

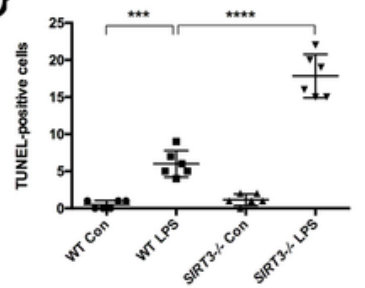

SIRT3-\%

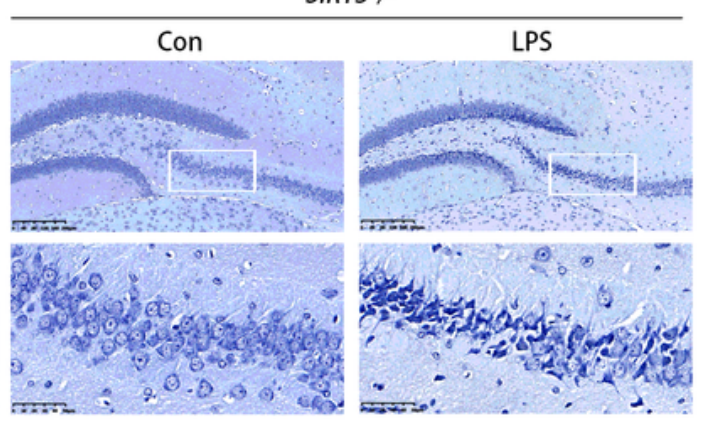

SIRT3-\%

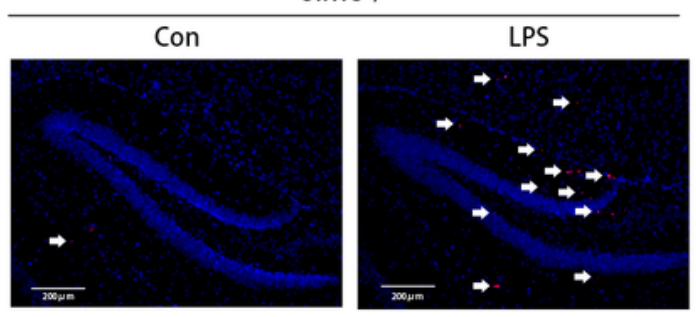

E
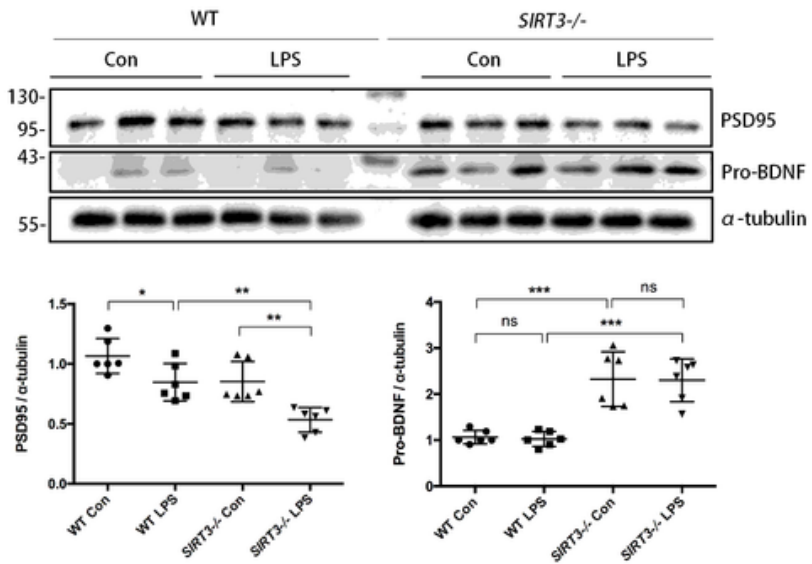

\section{Figure 3}

SIRT3 deficiency exacerbates hippocampal neuronal apoptosis and synaptic dysfunction in LPS-induced SAE. Representative images of Nissl's staining with zoomed in highlighted area (A) and TUNEL staining marked with white arrows of TUNEL-positive cells (B) in DG region $24 \mathrm{~h}$ after LPS exposure.

Quantification thickness of granule cell layer (C) and TUNEL-positive cells (D) in DG region. Each symbol represents one mouse $(n=6)$. Bars indicate the means \pm SD; P values were determined using ANOVA and 
Tukey's test for the post hoc analysis: ns, not significant; ${ }^{\star *} P<0.01 ; * \star \star P<0.001 ; * \star * * P<0.0001$. (E) Representative western blots of PSD95 and pro-BDNF in hippocampus. Relative protein levels were determined after normalization to a-tubulin. Each symbol represents one mouse $(n=6)$. Bars indicate the means \pm SD; $P$ values were determined using ANOVA and Tukey's test for the post hoc analysis: ns, not significant; $* P<0.05 ; * \star P<0.01 ; * \star * P<0.001$.
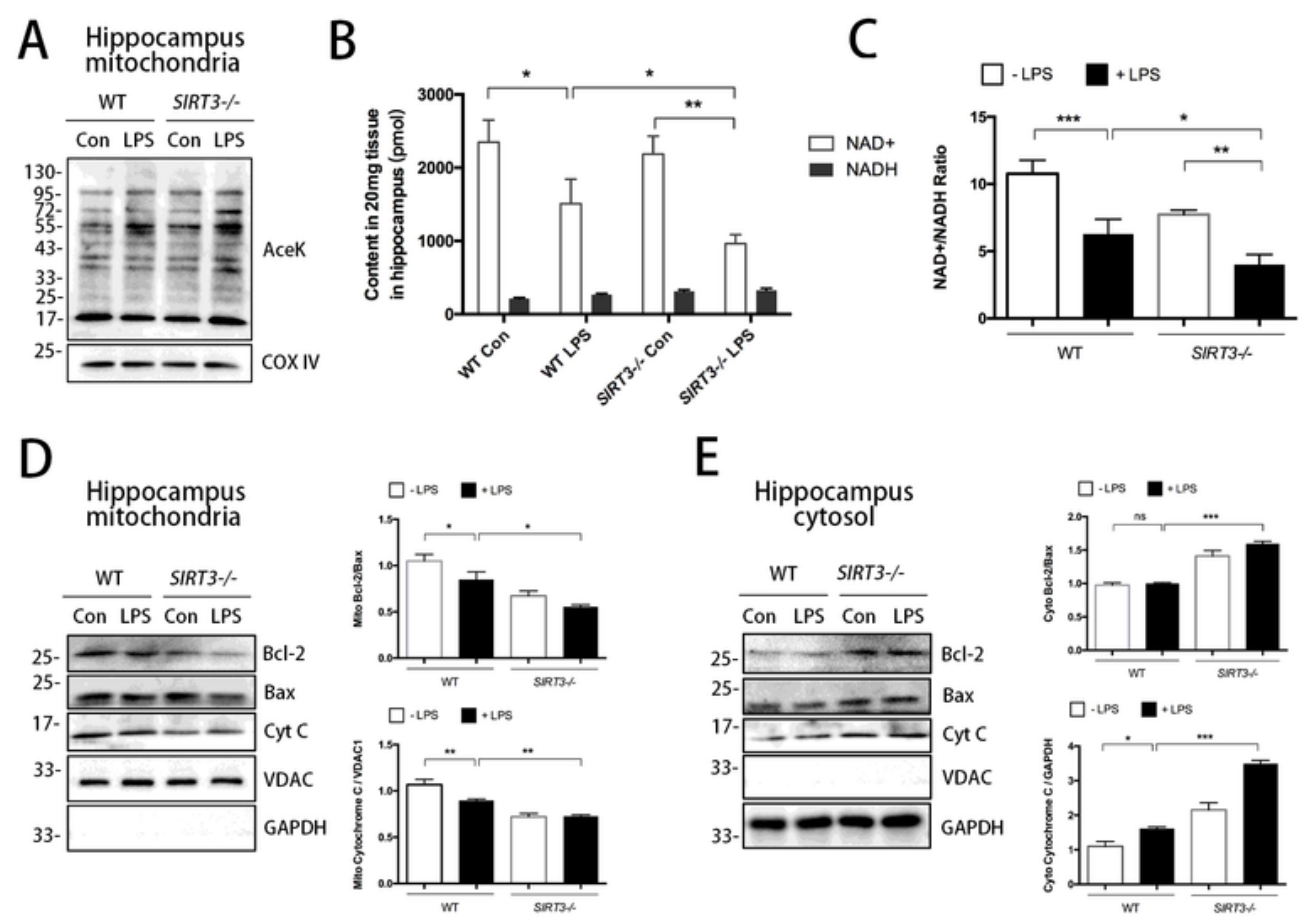

Figure 4 
SIRT3 deficiency decreased the hippocampal NAD+/NADH ratio and accelerated mitochondrial apoptosis in LPS-induced SAE. (A) Mitochondrial lysine acetylation level was assessed among the four groups $24 \mathrm{~h}$ after LPS exposure $(n=6)$. Hippocampal total NAD+, total NADH $(B)$ and NAD+/NADH ratio $(C)$ were assessed among the four groups $24 \mathrm{~h}$ after LPS exposure $(n=6)$. Bars indicate the means \pm SD; $P$ values were determined using ANOVA and Tukey's test for the post hoc analysis: ${ }^{*} P<0.05 ;{ }^{* \star P}<0.01 ;{ }^{* \star *} P<0.001$. Representative western blots of $\mathrm{Bcl}-2$, Bax and Cyt $\mathrm{C}$ in mitochondrial extracts (D) and cytosol extracts (E) in hippocampus $24 \mathrm{~h}$ after LPS exposure $(n=6)$. Bars indicate the means $\pm S D ; P$ values were determined using ANOVA and Tukey's test for the post hoc analysis: ${ }^{*} P<0.05 ;{ }^{* * P}<0.01 ; * \star * P<0.001$. 
A
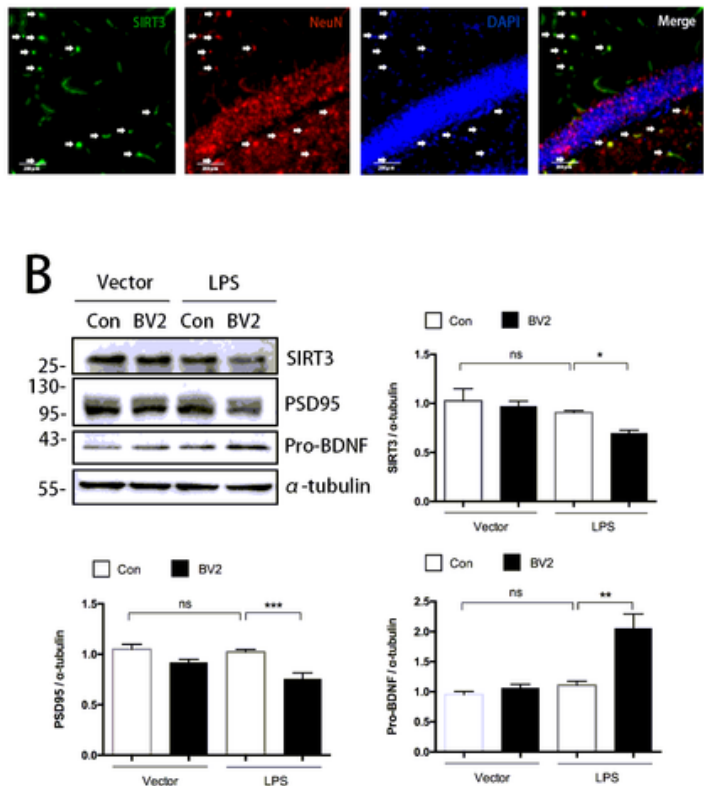

$\square \operatorname{con} a$

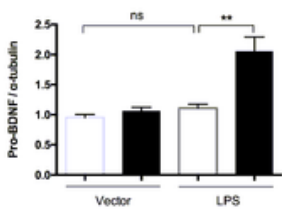

E
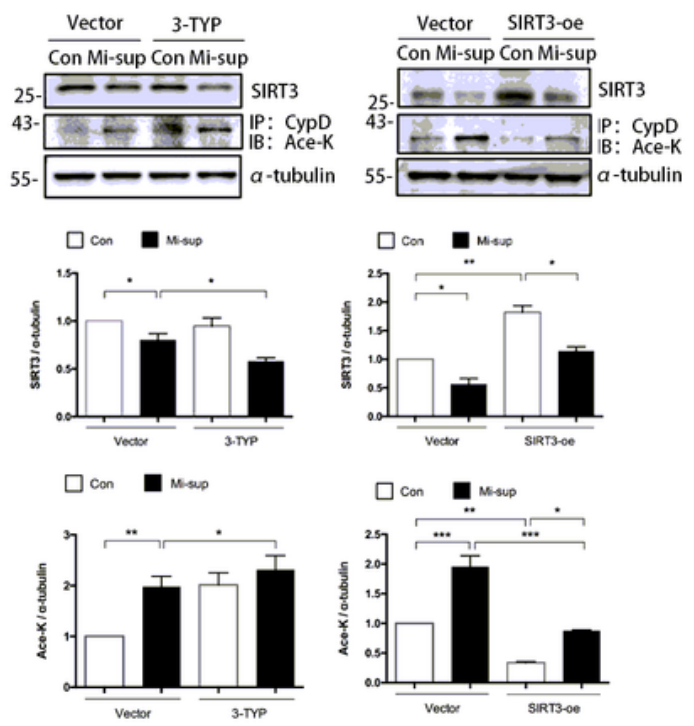

C

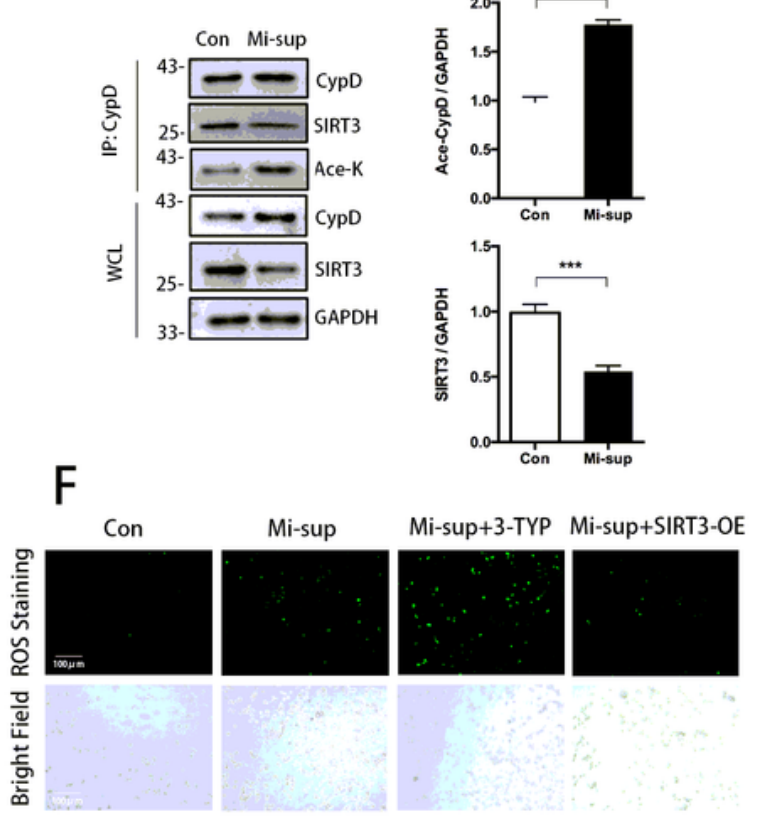

G

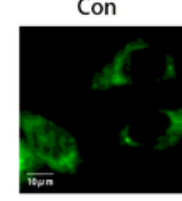

Mi-sup

Mi-sup+3-TYP Mi-sup+SIRT3-OE
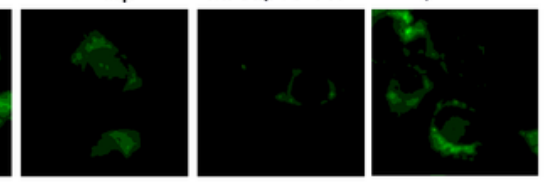

$\mathrm{H}_{\text {con }}$

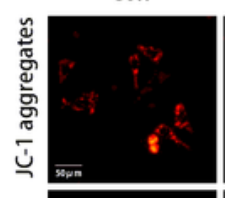

Mi-sup

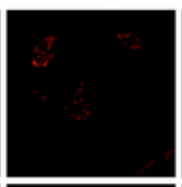

Mi-sup+3-TYP Mi-sup+SIRT3-OE
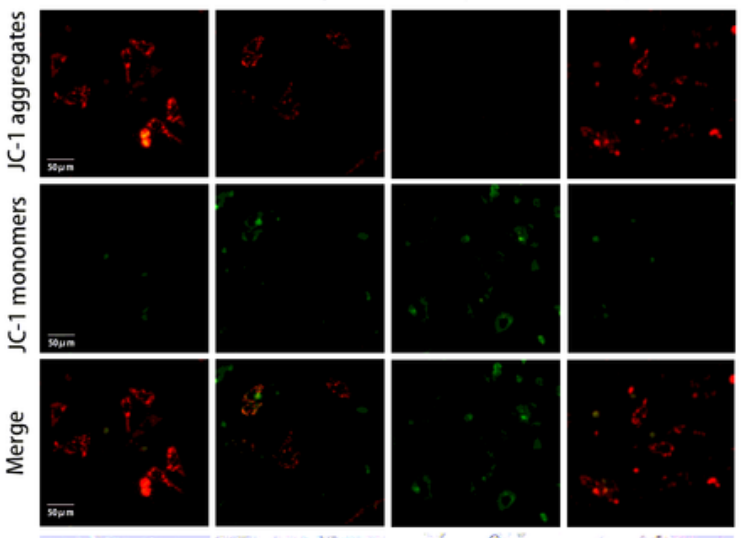

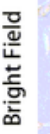

Q

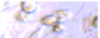

\section{Figure 5}

SIRT3-mediated deacetylation of CypD contributed to increased ROS generation, declined MMP and increased MPTP opening in HT22 cells with LPS-induced microglia supernatants. (A) The immunofluorescence staining was performed with SIRT3 (green), NeuN (red) and DAPI (blue) in mice hippocampus. The co-localization of SIRT3 and NeuN were marked with white arrows. (B) BV2 cells were first exposed to LPS $(1 \mu \mathrm{g} / \mathrm{ml})$ for $24 \mathrm{~h}$, and the supernatants of LPS-stimulated microglia (Mi-sup) were 
collected. HT22 cells were treated with LPS $(1 \mu \mathrm{g} / \mathrm{ml})$ or Mi-sup for $24 \mathrm{~h}$. Representative Western blots of SIRT3, PSD95 and pro-BDNF in HT22 whole cell lysates $(n=3)$. Bars indicate the means \pm SD; P values were determined using ANOVA and Tukey's test for the post hoc analysis: ns, not significant; ${ }^{*}<<0.05$; ${ }^{* \star P} P<0.01 ;{ }^{* \star *} P<0.001$. (C) HT22 cells were treated with Mi-sup for $24 \mathrm{~h}$. Whole cell lysates were prepared and probed in co-immunoprecipitation assays with an anti-CypD antibody. Western blot assays were performed for the immunoprecipitated samples with anti-SIRT3 and anti-Ace-K antibody $(n=3)$. Bars indicate the means $\pm S D ; P$ values were determined using t-test: $* \star \star P<0.001$. (D, E) HT22 cells were pretreated with 3-TYP $(50 \mu \mathrm{M})$ for $2 \mathrm{~h}$, or transfected with SIRT3 overexpression plasmid for $36 \mathrm{~h}$, then followed by Mi-sup treatment for $24 \mathrm{~h}$. Whole cell lysates were prepared and probed in coimmunoprecipitation assays with an anti-CypD antibody. Western blot assays were performed for the immunoprecipitated samples with anti-Ace-K antibody $(n=3)$. Bars indicate the means $\pm S D ; P$ values were determined using ANOVA and Tukey's test for the post hoc analysis: ${ }^{*} P<0.05 ;{ }^{* *} P<0.01 ;{ }^{* \star *} P<0.001$. (F) Representative images of intracellular ROS formation were measured by DCFH-DA. (G) MPTP was measured via calcein AM and $\mathrm{Co} 2+$ staining. $(\mathrm{H})$ Mitochondrial potential was measured via JC-1 staining. 
A $\begin{aligned} & \text { Hippocampus } \\ & \text { mitochondria }\end{aligned}$
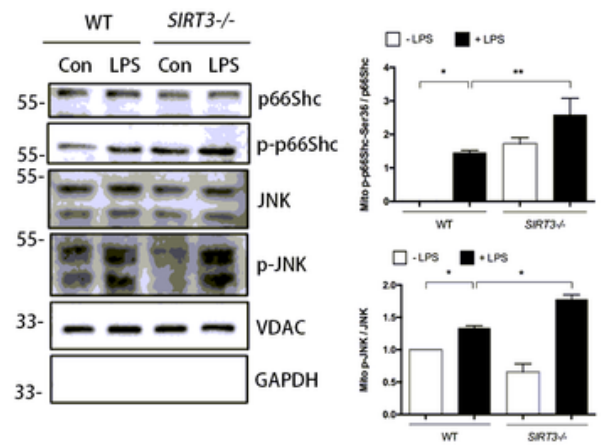

C $\underset{\text { mitochondria }}{\mathrm{HT} 22}$

$\frac{\text { Vector }}{\text { Con Mi-sup Con Mi-sup }}$

55- $---\infty$ p66Shc

p5- p-p66Shc

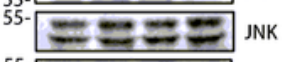

$55-30$ p-JNK

-

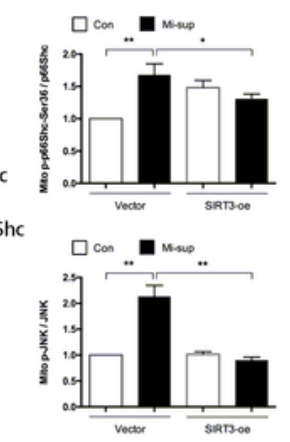

$\mathrm{E}$

HT22

mitochondria

Vector 3-TYP 3-TYP+SP600125

Con Mi-sup Con Mi-sup Con Mi-sup

55- p-p66She

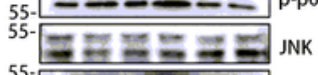

55-

33. ----1 VDAC con ano

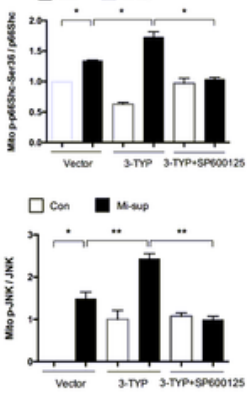

B

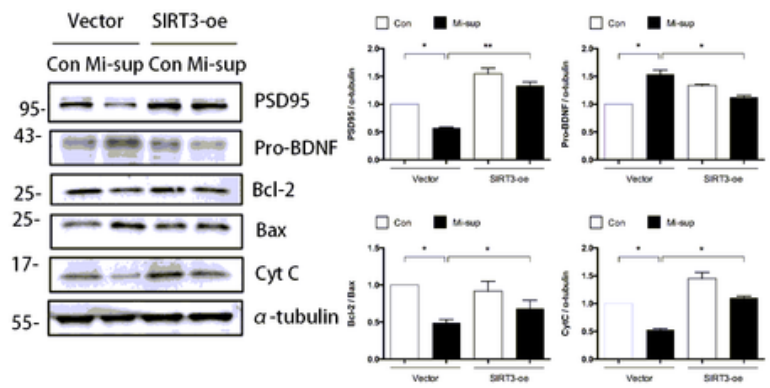

D

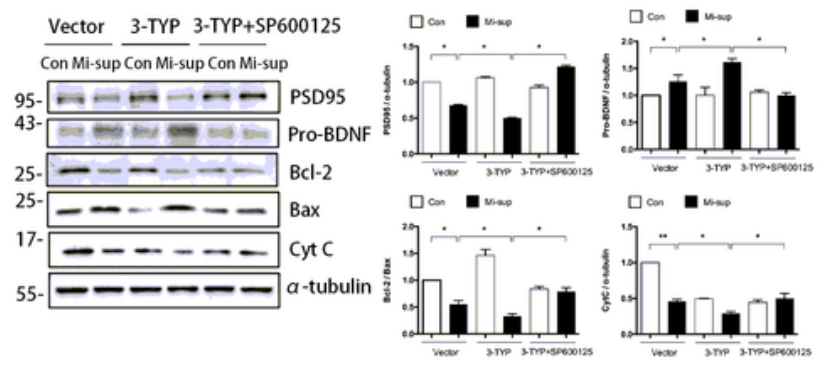

$\mathrm{F}$

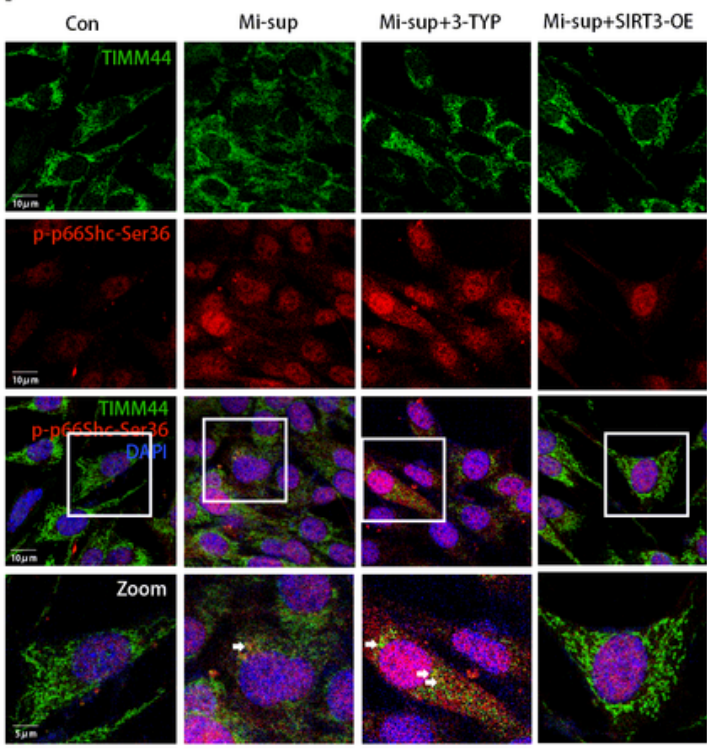

Figure 6

SIRT3 deacetylation activity contributed to synaptic dysfunction and mitochondrial apoptosis in HT22 cells with LPS-induced microglia supernatant via JNK/p66Shc pathway. (A) Representative western blots of p66Shc, p-p66Shc-Ser36, JNK and p-JNK in hippocampal mitochondrial extracts $24 \mathrm{~h}$ after LPS exposure $(n=6)$. Bars indicate the means \pm SD; $P$ values were determined using ANOVA and Tukey's test for the post hoc analysis: ${ }^{*}<<0.05 ;{ }^{*} \mathrm{P}<0.01$. HT22 cells were transfected with SIRT3 overexpression 
plasmid for $36 \mathrm{~h}$, then followed by Mi-sup treatment for $24 \mathrm{~h}$. Representative western blots of PSD95, proBDNF, Bcl-2, Bax and Cyt C in HT22 cells (B), and p66Shc, p-p66Shc-Ser36, JNK and p-JNK in HT22 mitochondrial extracts $(C)$. Bars indicate the means \pm SD; $P$ values were determined using ANOVA and Tukey's test for the post hoc analysis: ${ }^{*} \mathrm{P}<0.05 ;{ }^{*} \mathrm{P}<0.01$. HT22 cells were pretreated with 3-TYP $(50 \mu \mathrm{M})$ for $2 \mathrm{~h}$, or 3-TYP $(50 \mu \mathrm{M})$ and SP600125 $(30 \mu \mathrm{M})$ for $2 \mathrm{~h}$, then followed by Mi-sup treatment for $24 \mathrm{~h}$. Representative western blots of PSD95, pro-BDNF, Bcl-2, Bax and Cyt C in HT22 cells (D), and p66Shc, pp66Shc-Ser36, JNK and p-JNK in HT22 mitochondrial extracts (E). Bars indicate the means \pm SD; $P$ values were determined using ANOVA and Tukey's test for the post hoc analysis: ${ }^{*} P<0.05$; ${ }^{*} \mathrm{P}<0.01$. (F) Representative fluorescence images of HT22 cells with different treatment. From top to bottom: TIMM44 staining; p-p66Shc-Ser36 staining; overlay image of TIMM44 (green), p-p66Shc-Ser36 (red) and DAPI staining for nuclei (blue); zoom in of highlighted area in overlay images. The co-localization of TIMM44 and p-p66Shc-Ser36 were marked with white arrows. Scale bar, $10 \mu \mathrm{m}$, or $5 \mu \mathrm{m}$ for zoomed images. 